\title{
Fault-Tolerant Periodic Economic Model Predictive Control of Differential-Algebraic-Equation Systems
}

\author{
Ye Wang ${ }^{1}$, Vicenç Puig $^{1}$ and Gabriela Cembrano ${ }^{1,2}$
}

\begin{abstract}
This paper addresses a fault-tolerant periodic economic model predictive control (MPC) strategy for differentialalgebraic-equation (DAE) systems. Fault tolerance evaluation of the proposed economic MPC strategy uses set computations and a performance degradation analysis. By means of the set computations, the feasible solution set (including system states and control inputs) can be determined as well as the admissible performance set can be obtained when system reconfiguration or fault accommodation strategies are used. The proposed control strategy allows to carry out an analysis of performance degradation by using the feasible and admissible performance set. As a result, if the performance degradation is accepted, the economic MPC controller can be applied using system reconfiguration or fault accommodation. Finally, the proposed fault-tolerant MPC strategy is verified through an illustrative example.
\end{abstract}

\section{INTRODUCTION}

Fault-tolerant control (FTC) involves a variety of control theories, which maintain control systems in presence of faults running in a safe mode with an acceptable performance degradation. Especially in a safety-critical application, the use of FTC to adapt the control strategy in a faulty situation is mandatory. Generally speaking, there are two kinds of FTC techniques: active and passive. On the one hand, active FTC adapts the control loop taking into account the information from fault diagnosis. On the other hand, passive FTC considers the fault occurrences as system disturbances and makes control systems robust against faults. Thus, passive FTC can be also considered as an application of the robust control technique. Faults are usually taken into account as unknown but bounded in a known set.

Model predictive control (MPC) offers an alternative and effective framework for FTC [1], [2]. Fault-tolerant MPC allows adapting the configuration of the system model and constraints along the prediction horizon to take into account the fault effect. Recently, economic MPC (EMPC) has attracted a lot of attention. In contrast with classical MPC, EMPC aims at optimizing the economic system performance, rather than tracking the reference trajectory. The optimal control actions of EMPC are often found by means of an economic cost function that measures the economic system performance. Hence, the stage cost of EMPC is usually not

\footnotetext{
${ }^{1}$ Y. Wang, V. Puig and G. Cembrano are with Advanced Control Systems (SAC) Research Group at Institut de Robòtica i Informàtica Industrial, CSIC-UPC, Universitat Politècnica de Catalunya-BarcelonaTech, C/. Llorens i Artigas 4-6. 08028 Barcelona, Spain. E-mail: ywang@iri.upc.edu, vicenc.puig@upc.edu, cembranodiri.upc.edu

${ }^{2} \mathrm{G}$. Cembrano is also with CETaqua, Water Technology Centre, Ctra d'Esplugues 75, Cornellà de Llobregat, 08940 Barcelona, Spain.
}

in a quadratic form but in a time-varying manner depending on an exogenous signal.

Differential-algebraic-equation (DAE) systems, which are also known as singular or descriptor systems, have gained a lot of attention in many important applications, such as chemical processes [3], water systems [4] and electrical systems [5], [6]. It is known that the DAE system has two types of equations: differential and algebraic equations, where differential equations mainly describe the system dynamics and algebraic equations include the static relations between some elements (such as manipulated variables and algebraic variables) in the system.

This paper proposes a fault-tolerant periodic EMPC strategy for uncertain DAE systems. An on-line planner in the periodic EMPC strategy is used to produce the optimal steady states in the open loop. The fault tolerance evaluation of the EMPC controller is performed by means of a set computations. Therefore, the performance degradation is analyzed through computing the economic performance set after the faults occur. If the performance degradation is within the acceptable set, then the EMPC strategy can continue to be applied. A suitable fault diagnosis and identification (FDI) module is assumed to be available in this paper [7]. Depending on the information provided by the FTC system, two types of fault-tolerant mechanisms can be used: system reconfiguration and fault accommodation, both being considered in the proposed FTC methodology [8].

The remainder of this paper is structured as follows. In Section II, the periodic EMPC strategy for DAE systems with an on-line planner is presented. Fault-tolerant periodic EMPC strategy for DAE systems is proposed in Section III. In Section IV, the set-based approach for DAE systems is introduced. In Section V, an illustrative example is presented in order to verify the effectiveness of the proposed control strategy. Finally, some conclusions are drawn in Section VI.

Notations: The Minkowski sum of two sets $X$ and $Y$ is denoted by $X \oplus Y \triangleq\{x+y \mid x \in X, y \in Y\}$ and the Pontryagin difference of two sets $X$ and $Y$ is denoted by $X \ominus Y \triangleq\{x \in X \mid x+y \in X, y \in Y\}$. The inclusion operator between two sets $X$ and $Y$ is defined by $X \subseteq Y \triangleq\{x \mid$ if and only if $\forall x \in X$, then $x \in Y\}$. The intersection operator of two sets $X$ and $Y$ is defined by $X \cap Y \triangleq\{x \mid x \in X$ and $x \in Y\}$.

An $m$-order zonotope $\mathcal{Z} \in \mathbb{R}^{n}(m \geq n)$ is defined by a hypercube affine projection with the center $p \in \mathbb{R}^{n}$ and a matrix $H \in \mathbb{R}^{n \times m}$ as $\mathcal{Z}=p \oplus H \mathbf{B}^{m}$, where $\mathbf{B}^{m}$ denotes $m$-dimensional unitary box. Consider a zonotope $\mathcal{Z}=p \oplus H \mathbf{B}^{m}$, the smallest box (interval hull) containing the 
zonotope is given by $\square \mathcal{Z}=p \oplus r s(H) \mathbf{B}^{m}$, where $r s(H)$ is a diagonal matrix such that $r s(H)_{j}=\sum_{j=1}^{m}\left|H_{j}\right|$ with $H_{j}$ denoting the $j$-th column of the matrix $H$. Furthermore, the reduction operator for the zonotope proposed in [9] is denoted as $\downarrow_{q}$, where $q$ specifies the maximum number of column of segment matrix $H$.

\section{Periodic Economic Model Predictive Control FOR DAE SYSTEMS}

\section{A. Control-oriented Model}

Consider the discrete-time time-varying uncertain DAE system defined by

$$
\begin{aligned}
x(k+1) & =A(k) x(k)+B(k) u(k)+D(k) z(k)+D_{w}(k) \omega(k), \\
\mathbf{0} & =C_{x}(k) x(k)+C_{u}(k) u(k)+C_{z}(k) z(k)+C_{w}(k) \omega(k),
\end{aligned}
$$

where $x(k) \in \mathbb{X} \subseteq \mathbb{R}^{n_{x}}$ represents the vector of system states limited by the physical bounds $\mathbb{X}, u(k) \in \mathbb{U} \subseteq \mathbb{R}^{n_{u}}$ represents the vector of control inputs within physical limits $\mathbb{U}, z \in \mathbb{R}^{n_{z}}$ represents the vector of known exogenous periodic signals, $\omega(k) \in \mathbb{R}^{n_{w}}$ denotes the vector of system disturbances at time instant $k \in \mathbb{N}$, respectively. $A(k)$, $B(k), D(k), D_{w}(k), C_{x}(k), C_{u}(k)$ and $C_{z}(k)$ are timevarying system matrices of appropriate dimensions. (1a) is the discrete-time differential equation describing the system dynamics while (1b) is the discrete-time algebraic equation including the static relations.

The closed-loop DAE system is operated by an optimal periodic EMPC strategy. The performance of state evolutions is evaluated by economic stage cost function $\ell(k, x(k), u(k), p(k))$ that considers the system state, control input and an exogenous time-varying parameter $p(k)$. The economic parameter $p$ usually represents periodic cost, for instance, electricity price and its variation is assumed as known. Moreover, it is noted that the economic stage cost function $\ell(k, x(k), u(k), p(k))$ is not necessarily positive definite with respect to system states as it is in the classical tracking MPC [10], [11].

Assumption 1 (Continuity): The system states are continuous and the economic stage cost function $\ell(\cdot)$ are continuous and differentiable.

Definition 1: A DAE system is called T-periodic with respect to the exogenous periodic signal $z(k)=z(k+\mathrm{T})$ and parameter in economic stage cost function $p(k)=p(k+\mathrm{T})$ if there exists a period $\mathrm{T} \in \mathbb{Z}_{>1}$ such that for all $k \in \mathbb{N}$ it holds that $x(k+1)=x(k+\mathrm{T}+1)$ when the system disturbances are ignored. Thus, the following equations hold

$$
\begin{gathered}
A(k)=A(k+\mathrm{T}), B(k)=B(k+\mathrm{T}), \\
D(k)=D(k+\mathrm{T}), C_{x}(k)=C_{x}(k+\mathrm{T}), \\
C_{u}(k)=C_{u}(k+\mathrm{T}), C_{z}(k)=C_{z}(k+\mathrm{T}) .
\end{gathered}
$$

\section{B. EMPC Optimization Statement}

The periodic behavior implies that the time-varying economic stage cost function is T-periodic described by $\ell(k, x(k), u(k), p(k))=\ell(k+\mathrm{T}, x(k), u(k), p(k))$. The economic performance is measured with the average of the time-varying economic stage cost function of the closed loop as follows:

$$
L_{\infty}(0, x, u, p) \triangleq \lim _{\mathrm{T} \rightarrow \infty} \frac{1}{\mathrm{~T}} \sum_{i=0}^{\mathrm{T}-1} \ell(i, x(i), u(i), p(i)) .
$$

Assumption 2 (Robustness): The robustness of the EMPC controller can be realized by means of an appropriate constraint tightening technique (see [12], [13], [14] and [15]). Therefore, the tightened constraints on system states and control inputs can be formulated as follows:

$$
\begin{aligned}
& x(k) \in \mathcal{X}(k), \\
& u(k) \in \mathcal{U}(k) .
\end{aligned}
$$

The optimal steady state-input pair $\left(\mathbf{x}_{\infty}^{*}, \mathbf{u}_{\infty}^{*}\right)$ of the DAE system in (1) can be derived by solving the following infinitehorizon and open-loop optimization problem $\mathbb{P}_{\infty}^{s}$ with free initial state:

$$
\min _{x_{\infty}^{*}(i), u_{\infty}^{*}(i)} L_{\infty}(0, x, u, p)
$$

subject to

$$
\begin{aligned}
& x(i+1)=A(i) x(i)+B(i) u(i)+D(i) z(i), \\
& \mathbf{0}=C_{x}(i) x(i)+C_{u}(i) u(i)+C_{z}(i) z(i), \\
& x(i+1) \in \mathcal{X}^{r}(i+1), \\
& u(i) \in \mathcal{U}^{r}(i) .
\end{aligned}
$$

where $\mathcal{X}^{r}(i)$ and $\mathcal{U}^{r}(i)$ are subsets of $\mathcal{X}$ and $\mathcal{U}$ in order to ensure that the robust tightened constraints $\mathcal{X}$ and $\mathcal{U}$ have no influence on the system steady-state trajectory.

It is noticed that the above optimization problem considers an infinite horizon. Thus, from the application point of view, it is difficult and expensive to apply. However, considering the periodic behavior of the DAE system, the optimal steady trajectory can be obtained by a finite-horizon open-loop optimization problem $\mathbb{P}_{\mathrm{T}}^{s}$ with a periodic terminal constraint as follows:

$$
\min _{x_{s}^{*}(i), u_{s}^{*}(i)} L_{\mathrm{T}}^{s}(i, x, u, p) \triangleq \sum_{i=0}^{\mathrm{T}-1} \ell(i, x(i), u(i), p(i)),
$$

subject to

$$
\begin{aligned}
& x(i+1)=A(i) x(i)+B(i) u(i)+D(i) z(i), \\
& \mathbf{0}=C_{x}(i) x(i)+C_{u}(i) u(i)+C_{z}(i) z(i), \\
& x(i+1) \in \mathcal{X}^{r}(i+1), \\
& u(i) \in \mathcal{U}^{r}(i), \\
& x(0)=x(\mathrm{~T}) .
\end{aligned}
$$

Remark 1: The optimization problems $\mathbb{P}_{\infty}^{s}$ and $\mathrm{P}_{\mathrm{T}}^{s}$ are only solved once. The solutions are regarded as the optimal periodic steady states and control inputs.

The feasible solutions of the optimization problem $\mathbb{P}_{\mathrm{T}}^{s}$ denoted $\mathbf{x}_{s}^{*}=\left\{x_{s}^{*}(1), \ldots, x_{s}^{*}(\mathrm{~T})\right\}$, $\mathbf{u}_{s}^{*}=\left\{u_{s}^{*}(0), u_{s}^{*}(1), \ldots, u_{s}^{*}(\mathrm{~T}-1)\right\}$ are regarded as the best feasible periodic steady-state pair $\left(\mathbf{x}_{s}^{*}, \mathbf{u}_{s}^{*}\right)$.

Lemma 1 (The Equivalent Solutions): The solutions of the optimization problems $\mathbb{P}_{\infty}^{s}$ and $\mathbb{P}_{\mathrm{T}}^{s}$ satisfy the following conditions:

$$
\begin{aligned}
\mathbf{x}_{s}^{*} & =\mathbf{x}_{\infty}^{*}, \\
\mathbf{u}_{s}^{*} & =\mathbf{u}_{\infty}^{*}, \\
L_{\mathrm{T}}^{s}\left(i, \mathbf{x}_{s}^{*}, \mathbf{u}_{s}^{*}, p\right) & =\mathrm{T} L_{\infty}\left(0, \mathbf{x}_{\infty}^{*}, \mathbf{u}_{\infty}^{*}, p\right) .
\end{aligned}
$$


Proof: The proof can be found in [16].

Following the single-layer EMPC controller proposed in [16], the steady state-input pair $\left(\mathbf{x}_{s}^{*}, \mathbf{u}_{s}^{*}\right)$ can be found in an on-line optimization planner as $\mathbb{P}_{\mathrm{T}}^{s}$. The cost function includes two parts: (1) An economic term for the prediction model with the enlarged prediction horizon as proposed in [4] that governs the economic transient behavior. (2) An economic term in the planner that measures the economic performance. Therefore, this cost function is formulated as

$$
\begin{aligned}
L_{\mathrm{T}}^{e}(k, x, u, p) \triangleq & \sum_{i=0}^{n \mathrm{~T}-1} \ell(i, x(i \mid k), u(i \mid k), p(i)) \\
& +L_{\mathrm{T}}^{s}\left(i, x^{p}, u^{p}, p\right),
\end{aligned}
$$

where $x^{p}$ and $u^{p}$ denote the predicted state and input of the on-line EMPC planner, respectively.

In general, the periodic economic MPC strategy for DAE systems can be formulated by solving the following optimization problem $\mathbb{P}_{\mathrm{T}}^{e}(k)$ :

$$
\min _{x^{*}(k), u^{*}(k)} L_{\mathrm{T}}^{e}(k, x, u, p)
$$

subject to

$$
\begin{aligned}
& x(i+1 \mid k)=A(k+i) x(i \mid k)+B(k+i) u(i \mid k)+D(k+i) z(i \mid k), \\
& \mathbf{0}=C_{x}(k+i) x(i \mid k)+C_{u}(k+i) u(i \mid k)+C_{z}(k+i) z(i \mid k), \\
& x(i+1 \mid k) \in \mathcal{X}(i+1 \mid k) \\
& u(i \mid k) \in \mathcal{U}(i \mid k) \\
& x(\mathrm{~T} \mid k)=x^{p}(\mathrm{~T}) \\
& x(0 \mid k)=x(k) \\
& x^{p}(i+1)=A(i) x^{p}(i)+B(i) u^{p}(i)+D(i) z(i) \\
& \mathbf{0}=C_{x}(i) x^{p}(i)+C_{u}(i) u^{p}(i)+C_{z}(i) z(i) \\
& x^{p}(i+1) \in \mathcal{X}^{r}(i+1) \\
& u^{p}(i) \in \mathcal{U}^{r}(i) \\
& x^{p}(0)=x^{p}(\mathrm{~T})
\end{aligned}
$$

where constraints (12b)-(12g) represents the prediction model of the DAE system. Constraints (12h)-(12l) denote the on-line planner that is able to find the steady state $x^{p}(\mathrm{~T})$ that is subsequently set as the terminal state as the prediction model of the DAE system.

After solving the above optimization problem $\mathbb{P}_{T}^{e}(k)$, the optimal control law is determined by the receding horizon approach such that the first value of a sequence of control inputs $u^{*}(0 \mid k), \ldots, u^{*}\left(\mathrm{H}_{\mathrm{p}}-1 \mid k\right)$ is the optimal control action at current time instant:

$$
u(k) \triangleq u^{*}(0 \mid k)
$$

\section{FAUlT-TOLERANT MEChANiSMS FOR ECONOMIC Model PREdictive CONTROL}

\section{A. Fault-tolerant Mechanisms}

FTC can be included in the EMPC strategy presented in the previous section in two ways depending of the information provided by the FDI block:
1) System Reconfiguration: The first strategy to adapt the control loop to the fault presence, known as system reconfiguration, is based on changing the control law and other elements of the closed-loop as required. This strategy could be applied in case the FDI module does not provide any information about the fault estimation. In this case, faulty components are unplugged by the supervisory system and the control objectives should be achieved using the non-faulty components. In case that the faults appeared in a given set, the control-oriented model of DAE systems in (1) is modified as follows:

$$
\begin{aligned}
x(k+1) & =A(k) x(k)+\sum_{i \in I_{N}} B_{i}(k) u_{i}(k)+D(k) z(k)+D_{w}(k) \omega(k), \\
\mathbf{0} & =C_{x}(k) x(k)+\sum_{i \in I_{N}} C_{u, i}(k) u_{i}(k)+C_{z}(k) z(k)+C_{w}(k) \omega(k),
\end{aligned}
$$

where $I_{N}$ denotes the subset of healthy actuators.

2) Fault Accommodation: Fault accommodation involves the estimation of actual system constraints and parameters provided by the FDI module. By means of a suitable fault diagnosis method, the control inputs are modified while the rest of the elements within the control loop are kept unchanged. After the faults occurred, the control-oriented model of DAE systems in (1) is modified as follows:

$$
\begin{aligned}
x(k+1)= & A(k) x(k)+\sum_{i \in I_{N}} B_{i}(k) u_{i}(k)+\sum_{j \in I_{F}} \Phi_{j}\left(k, u_{j}(k), \theta_{f}\right) \\
& +D(k) z(k)+D_{w}(k) \omega(k), \\
\mathbf{0}= & C_{x}(k) x(k)+\sum_{i \in I_{N}} C_{u, i}(k) u_{i}(k)+\sum_{j \in I_{F}} \Psi_{j}\left(k, u_{j}(k), \theta_{f}\right) \\
& +C_{z}(k) z(k)+C_{w}(k) \omega(k),
\end{aligned}
$$

where $I_{F}$ denotes the subset of faulty actuators. $\Phi_{j}(\cdot)$ and $\Psi_{j}(\cdot)$ denote the mapping functions estimated by the FDI module. $\theta_{f}$ denotes the parameter depending on the estimated faults. Note that this parameter can be estimated by using the set-membership strategy proposed in [7] and the multiple model (including faulty and healthy models) method proposed in [17].

\section{B. Fault-tolerant Periodic Economic MPC for DAE Systems}

Taking into account the two types of fault-tolerant mechanisms presented in previous subsection, the fault-tolerant EMPC strategy for periodic DAE systems can be implemented by solving the following optimization problem $\mathrm{P}_{\mathrm{T}}^{f}(k)$ :

$$
\min _{x^{*}(k), u^{*}(k)} L_{\mathrm{T}}^{e}(k, x, \widetilde{u}, p),
$$


subject to

$$
\begin{aligned}
& x(i+1 \mid k)=A(k+i) x(i \mid k)+\widetilde{B}(k+i) \widetilde{u}(i \mid k)+D(k+i) z(i \mid k), \\
& \mathbf{0}=C_{x}(k+i) x(i \mid k)+\widetilde{C}_{u}(k+i) \widetilde{u}(i \mid k)+C_{z}(k+i) z(i \mid k), \\
& x(i+1 \mid k) \in \mathcal{X}(i+1 \mid k), \\
& u(i \mid k) \in \mathcal{U}(i \mid k), \\
& x(\mathrm{~T} \mid k)=x^{p}(\mathrm{~T}), \\
& x(0 \mid k)=x(k) \\
& x^{p}(i+1)=A(i) x^{p}(i)+\widetilde{B}(i) \widetilde{u}^{p}(i)+D(i) z(i), \\
& \mathbf{0}=C_{x}(i) x^{p}(i)+\widetilde{C}_{u}(i) \widetilde{u}^{p}(i)+C_{z}(i) z(i), \\
& x^{p}(i+1) \in \mathcal{X}^{r}(i+1), \\
& u^{p}(i) \in \mathcal{U}^{r}(i) \\
& x^{p}(0)=x^{p}(\mathrm{~T})
\end{aligned}
$$

where $\widetilde{B}, \widetilde{C}_{u}, \widetilde{u}$ and $\widetilde{u}^{p}$ are associated with system configuration or fault accommodation according to the information provided by the FDI module. After the prediction model is modified, the planner model is also needed to be adapted since the equilibrium point of the DAE system is changed.

\section{SET-BASEd ApProACH FOR FTC EVAluation of DAE SYSTEMS}

In this approach, the prediction model of the DAE system with the system state feedback is considered. The procedure of set-based approach for DAE systems mainly includes two steps as shown in Figure 1: A forward propagation, in which the direct image is built by using the differential equation (1a) with the Minkowski sum operator. Then, this compact set intersects with the polyhedral set built by algebraic equation (1b) and the constraints on states $\mathcal{X}$ in a polyhedral form as $\mathcal{P}_{x}$. A backward propagation, in which the inverse image is built by using the transformed algebraic equation based on (1b) with Pontryagin difference operator.

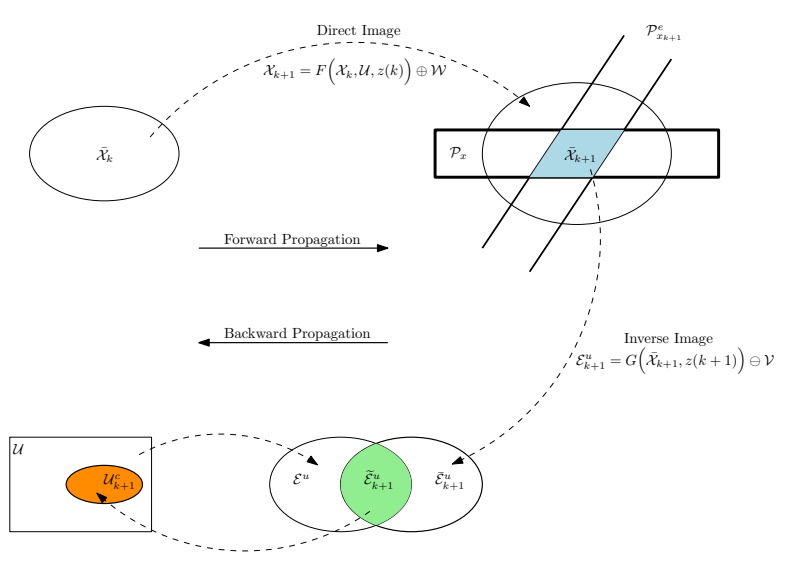

Fig. 1. Topology of Forward and Backward Propagation

System disturbance $\omega(k+i)$ is bounded by a known zonotpic set with the center $\omega^{c}$ and segment matrix $H_{\omega}$ as

$$
\omega(k+i) \in \mathcal{W} \triangleq \omega^{c} \oplus H_{\omega} \mathbf{B}^{n_{\omega}}, \quad i \in \mathbb{Z}_{[0, T-1]} .
$$

\section{A. Set Definitions}

Before presenting the procedure of the calculations, some necessary definitions are introduced as follows:
Definition 2 (Uncertain Dynamic and Static States Sets): Consider a DAE system (1), the uncertain dynamic states set $\mathcal{X}_{k}^{d}$ in (1a) is approximated by a zonotopic set and the uncertain static states set $\mathcal{X}_{k}^{s}$ in (1b) is approximated by a polyhedron set.

Definition 3 (Consistent States Set): Given a uncertain DAE system (1) containing system dynamics (1a) and static relations (1b), the consistent states set $\overline{\mathcal{X}}_{k}$ at time instant $k$ is defined by intersections of uncertain dynamic states set, static states set and admissible states set as $\overline{\mathcal{X}}_{k}=\mathcal{X}_{k}^{d} \cap \mathcal{X}_{k}^{s} \cap \mathcal{X}(k)$.

Definition 4 (Compatible Inputs Set): Given a DAE system (1), the compatible input set at time instant $k$ is defined as $\mathcal{U}_{k}^{c}=\mathcal{U}(k) \ominus \mathcal{U}_{k}^{r}$, where $\mathcal{U}_{k}^{r}$ denotes the reverse incompatible inputs set that can be found through backward propagation.

Definition 5 (Feasible Solutions Set): The feasible solutions set at time instant $k$ is given by

$$
\Omega_{k} \triangleq\left\{(x(k), u(k)) \mid x(k) \in \overline{\mathcal{X}}_{k}, u(k) \in \mathcal{U}_{k}^{c},\right\},
$$

and corresponds to the input and state sets compatible with system constraints.

Definition 6 (Economic Performances Set): The feasible economic performances set along the MPC prediction horizon is given by

$$
\mathcal{J}_{\Omega_{k}} \triangleq\left\{\ell(k, x(k), u(k), p(k)) \in \mathbb{R} \mid(x(k), u(k)) \in \Omega_{k}\right\},
$$

and corresponds to the set of all values of $\mathcal{J}_{\Omega_{k}}$ obtained from feasible solutions set $\Omega_{k}$.

Definition 7 (Admissible Economic Performances Set):

The nominal economic performance of the EMPC controller can be computed taking into account the steady state-input pair $\left(\mathbf{x}_{s}^{*}, \mathbf{u}_{s}^{*}\right)$, which is given by

$$
\mathcal{J}_{A_{k}}^{c} \triangleq \ell\left(k, x_{s}^{*}(k), u_{s}^{*}(k), p(k)\right),
$$

and consider a tolerance interval $\mathcal{F}_{e}$, then the admissible economic performance set is defined as

$$
\mathcal{J}_{A_{k}} \triangleq \mathcal{J}_{A_{k}}^{c} \oplus \mathcal{F}_{e}
$$

It is noted that the admissible economic performance set can be changed when one of fault-tolerant mechanisms is performed.

\section{B. Forward Propagation}

From the last state measurement $x_{k-1}$, the set-based approach for DAE systems is initialized. All the admissible states can be included in a compact zonotopic set $\mathcal{X}_{k}=$ $\mathcal{X}_{k}^{c} \oplus H_{\mathcal{X}_{k}} \mathbf{B}^{n_{x} \times r_{x}}$ and physical control input set $\mathbb{U}$ can be also represented as a zonotope $\mathbb{U}=u^{c} \oplus H_{u} \mathbf{B}^{n_{u} \times r_{u}}$. The MPC prediction horizon is selected as the propagation horizon. In forward propagation, the uncertain state set is firstly computed as a zonotopic set by means of dynamic equation (1a) as follows:

$$
\begin{aligned}
\mathcal{X}_{k}^{c}= & A(k-1) x(k-1)+B(k-1) u^{c} \\
& +D(k-1) z(k-1)+D_{w}(k-1) \omega^{c}, \\
H_{\mathcal{X}_{k}}= & {\left[B(k-1) H_{u}, D_{w}(k-1) H_{\omega}\right] . }
\end{aligned}
$$

The zonotopic sets can be represented by polyhedral sets in half-space form. The above zonotopic uncertain state set can 
be transformed into a polyhedral set after using a reduction operator to obtain $\downarrow_{q}\left(H_{\mathcal{X}_{k}}\right)$ proposed in [9] as follows:

$$
\mathcal{P}_{x_{k}}^{d} \triangleq\left\{x(k) \in \mathbb{R}^{n_{x}} \mid Q_{x}(k) x(k) \leq \mathbf{1}\right\}
$$

Besides, the polyhedral uncertain state set at time instant $k$ based on the algebraic equation (1b) can be obtained by

$$
\mathcal{P}_{x_{k}}^{e} \triangleq\left\{x(k) \in \mathbb{R}^{n_{x}} \mid \mathbf{C}_{x}(k) x(k) \leq \mathbf{P}_{x}^{0}(k)\right\},
$$

where

$$
\begin{aligned}
\mathbf{C}_{x}(k) & =\left[\begin{array}{c}
C_{x}(k) \\
-C_{x}(k)
\end{array}\right], \\
\mathbf{P}_{x}^{0}(k) & =\left[\begin{array}{c}
C_{u} H_{u}+C_{w}(k) H_{\omega}-E_{c}(k) \\
C_{u} H_{u}+C_{w}(k) H_{\omega}+E_{c}(k)
\end{array}\right], \\
E_{c}(k) & =C_{u} u^{c}+C_{z} z(k) .
\end{aligned}
$$

Then the consistent state set $\overline{\mathcal{X}}_{k}$ at time instant $k$ can be obtained by the following intersections:

$$
\overline{\mathcal{X}}_{k} \triangleq \mathcal{P}_{x_{k}}^{d} \cap \mathcal{P}_{x_{k}}^{e} \cap \mathcal{P}_{x} .
$$

For the step $k+i$ with $i=1,2, \ldots, N$, the calculation is similar but with a slight difference. Firstly, the zonotopic uncertain state set is given as

$$
\begin{aligned}
\mathcal{X}_{k+i}^{c}= & A(k+i-1) \overline{\mathcal{X}}_{k+i-1}+B(k+i-1) u^{c} \\
& +D(k+i-1) z(k+i-1)+D_{w}(k+i-1) \omega^{c}, \\
H_{\mathcal{X}_{k+i}}= & {\left[A(k+i-1) \downarrow_{q}\left(H_{\mathcal{X}_{k+i-1}}\right), B(k+i-1) H_{u}\right.} \\
& \left.D_{w}(k+i-1) H_{\omega}\right] .
\end{aligned}
$$

With the reduction of segment matrix $H_{\mathcal{X}_{k+i}}$, the polyhedral representation of the uncertain state set is denoted by

$$
\mathcal{P}_{x_{k+i}}^{d} \triangleq\left\{x(k+i) \in \mathbb{R}^{n_{x}} \mid Q_{x}(k+i) x(k+i) \leq \mathbf{1}\right\} .
$$

In terms of the polyhedral uncertain state set based on algebraic equation (1b), it is noticed that the system disturbance $\omega_{k}$ has to be accumulated for the multiple propagation. Thus, the polyhedral uncertain state set at time instant $k+i$ by using algebraic equation (1b) can be computed as follows:

$$
\mathcal{P}_{x_{k+i}}^{e} \triangleq\left\{x(k+i) \in \mathbb{R}^{n_{x}} \mid \mathbf{C}_{x}(k+i) x_{k+1} \leq \mathbf{P}_{x}(k+i)\right\},
$$

where

$$
\begin{aligned}
\mathbf{C}_{x}(k+i) & =\left[\begin{array}{c}
C_{x}(k+i) \\
-C_{x}(k+i)
\end{array}\right], \\
\mathbf{P}_{x}(k+i) & =\left[\begin{array}{c}
C_{u} H_{u}+E_{\omega}(k+i)-E_{c}(k+i) \\
C_{u} H_{u}+E_{\omega}(k+i)+E_{c}(k+i)
\end{array}\right], \\
E_{\omega}(k+i) & =C_{w}(k+i) H_{\omega} . \\
E_{c}(k+i) & =C_{u} u^{c}+C_{z} z(k+i) .
\end{aligned}
$$

The consistent state set $\overline{\mathcal{X}}_{k+i}$ at time instant $k+i$ can be computed as

$$
\overline{\mathcal{X}}_{k+i}=\mathcal{P}_{x_{k+i}}^{d} \cap \mathcal{P}_{x_{k+i}}^{e} \cap \mathcal{P}_{x} .
$$

After repeating calculations until time instant $k+N$, a series of consistent state sets along the horizon $N$ can be obtained. Figure 2 shows sampled consistent state sets $\overline{\mathcal{X}}_{k+i}$ of the example in Section $\mathrm{V}$ in the magenta polyhedron.

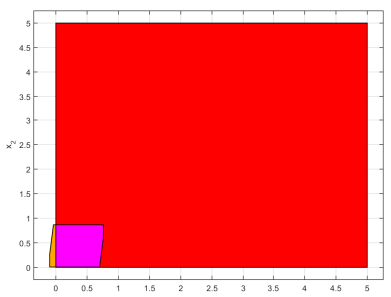

(a) $i=0$

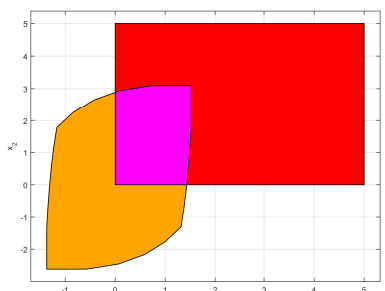

(c) $i=5$

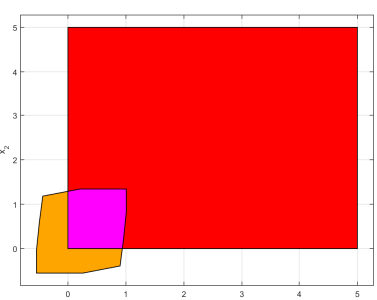

(b) $i=1$

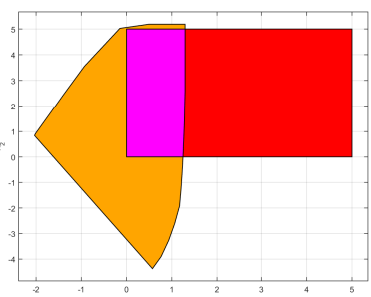

(d) $i=10$
Fig. 2. Sampled consistent states sets obtained through forward propagations: red box denotes the physical limitation of states $\mathcal{P}_{x}$, orange polyhedron denotes the possible uncertain region of states at time instant $k$ and magenta polyhedron denotes the consistent state set $\overline{\mathcal{X}}_{k}$ at time instant $k$.

\section{Backward Propagation}

As shown in Figure 1, the compatible input set $\mathcal{U}_{k}^{c}$ is difficult to obtain straightforwardly, because the inverse operator of the Minkowski sum is not easy to implement. Hence, a projection hyperplane is found by using the $C_{u}$-multiplier. Then, this hyperplane is denoted as $\mathcal{E}^{u}=C_{u} \mathbb{U}$. The inverse image at time instant $k+j$ with $j=N, N-1, \ldots, 1$ can be mathematically formulated as follows:

$$
\overline{\mathcal{E}}_{k+j}^{u} \triangleq \overline{\mathcal{X}}_{k+j} \ominus C_{z} z(k+j) \ominus \mathcal{W} .
$$

The compatible input set $\widetilde{\mathcal{E}}_{k+j}^{u}$ at time instant $k+j$ can be obtained by the intersection between the inverse image and the projection of the physical limitation on control input as follows:

$$
\widetilde{\mathcal{E}}_{k+j}^{u} \triangleq \overline{\mathcal{E}}_{k+j}^{u} \cap \mathcal{E}^{u} .
$$

The set $\widetilde{\mathcal{E}}_{k+j}^{u}$ can be represented in a polyhedral form:

$$
\widetilde{\mathcal{E}}_{k+j}^{u} \triangleq\left\{u(k+j) \in \mathbb{R}^{n_{u}} \mid Q_{u}(k+j) C_{u}(k+j) u(k+j) \leq \mathbf{1}\right\} .
$$

Then, the compatible control input set $\mathcal{U}_{k+j}^{c}$ can be obtained by

$$
\mathcal{U}_{k+j}^{c}=\left\{u(k+j) \mid u(k+j) \in \mathcal{U}(k+j), u(k+j) \in \widetilde{\mathcal{E}}_{k+j}^{u}\right\} .
$$

\section{Economic Performance Degradation Analysis}

After performing the forward and backward propagation, the feasible solutions set in Definition 5 can be obtained at each time instant. Then, the economic performance set in Definition 6 can be computed by the direct image from the economic stage function $\ell(k, x(k), u(k))$.

Given the admissible economic performance set $\mathcal{J}_{A_{k}}$, the analysis of the degradation performance can be proceed as shown in Fig. 3. If $\mathcal{J}_{\Omega_{k}} \cap \mathcal{J}_{A} \neq \varnothing$ in Fig. 3(a), then the 
closed-loop system can continue running after some faults occurred. If $\mathcal{J}_{\Omega_{k}} \cap \mathcal{J}_{A}=\varnothing$ in Fig. 3(b), then the admissible economic performance cannot be achieved. Hence, it may be necessary to add new actuators to the system in order to increase the fault-tolerance.

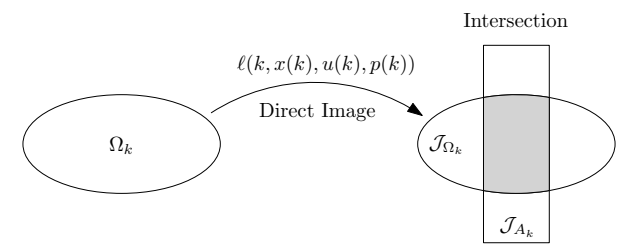

(a) Admissible Performance

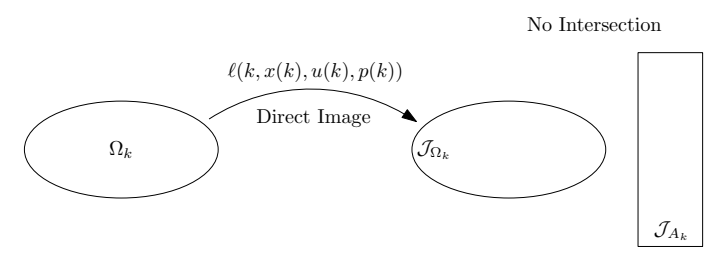

(b) Non-admissible Performance

Fig. 3. Economic Performance Analysis

As shown in Algorithm 1, the analysis of the economic performance with periodically admissible economic performance set $\mathcal{J}_{A_{k}}$ is performed.

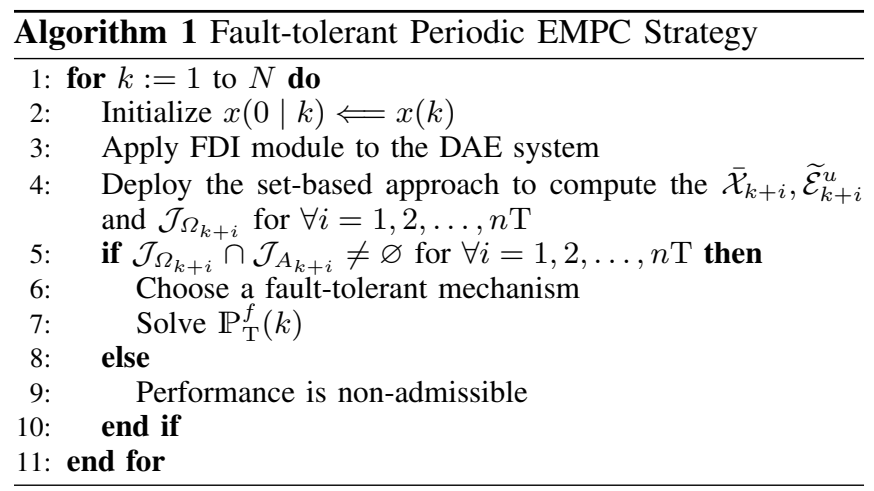

\section{ILlustrative EXAMPLE}

In order to illustrate the proposed fault-tolerant EMPC strategy, a linear discrete-time DAE system is considered as follows:

$$
\begin{aligned}
x(k+1) & =A(k) x(k)+B u(k)+D z(k)+D_{w} \omega(k), \\
\mathbf{0} & =C_{x} x(k)+C_{u} u(k)+C_{z} z(k)+C_{w} \omega(k),
\end{aligned}
$$

where

$$
\begin{aligned}
A(k) & =\left[\begin{array}{ll}
0.8 & 0 \\
0.2 & 1
\end{array}\right]+0.1 \sin (t), \\
B & =\left[\begin{array}{ccc}
1 & 0 & 0.1 \\
0 & 0.01 & 1
\end{array}\right], \\
D & =\left[\begin{array}{cc}
-1 & 0 \\
0 & -1
\end{array}\right], D_{w}=\mathbf{0}, \\
C_{x} & =\left[\begin{array}{ll}
1 & -1
\end{array}\right], C_{u}=\left[\begin{array}{lll}
1 & 1 & 1
\end{array}\right], \\
C_{z} & =\left[\begin{array}{ll}
-1 & -1
\end{array}\right], C_{w}=\mathbf{0},
\end{aligned}
$$

The period of the DAE system is $\mathrm{T}=24$. The MPC prediction horizon is chosen as the period $T$, that is $n=1$ in (11). The initial state vector is given as $x_{0}=[0.8,0.6]^{T}$. The economic stage cost function is defined as

$$
\ell(k, x(k), u(k), p(k))=\lambda_{1} x_{1}(k)+\lambda_{2} p(k)^{T} u(k),
$$

where $\lambda_{1}$ and $\lambda_{2}$ denote the weights for each objective. In the simulations, the following values are used: $\lambda_{1}=0.01$ and $\lambda_{2}=1$.

The set of the admissible economic performances is defined as

$$
\mathcal{J}_{A_{k}}=\left\{J \in \mathbb{R} \mid \mathcal{J}_{A_{k}}^{c} \leq J \leq \mathcal{J}_{A_{k}}^{c}+0.45\right\},
$$

where the performance degradation is decided as prior by evaluating the system redundancy.

Considering the two FTC strategies introduced before, the following scenarios will be considered for illustrative purposes:

- System Reconfiguration: actuator $u_{2}(k)$ is in faulty situation at time instant 48 and then this actuator is removed since this step

$$
\begin{aligned}
\widetilde{B} & =\left[\begin{array}{cc}
1 & 0.1 \\
0 & 1
\end{array}\right], \\
\widetilde{C}_{u} & =\left[\begin{array}{ll}
1 & 1
\end{array}\right],
\end{aligned}
$$

with $\widetilde{u}=\left[u_{1}, u_{3}\right]^{T}$. The simulation result is shown in Fig. 4. The system is running in a healthy mode at the beginning. From the dash line, there is a fault in actuator $u_{2}$. Through the system reconfiguration, the periodic steady-state trajectory is updated by the online planner. With the economic performance analysis, the admissible performance is accepted and then the system can continue running.

- Fault Accommodation: actuator $u_{2}(k)$ partially malfunctioned at time instant 48 . Through a suitable fault estimation module, the system model is modified as

$$
\begin{aligned}
\widetilde{B} & =\left[\begin{array}{ccc}
1 & 0 & 0.1 \\
0 & 0.02 & 1
\end{array}\right], \\
\widetilde{C}_{u} & =\left[\begin{array}{lll}
1 & 0.2 & 1
\end{array}\right],
\end{aligned}
$$

with $\widetilde{u}(k)=u(k)$. The simulation is shown in Fig. 5 . Similar to the previous case, the periodic steady-state trajectory is changed by removing partial performance of the faulty actuator. Since Algorithm 1 is applied, if the economic performance is admissible, the system can continue running.

The costs of two cases are plotted in Fig. 6. From this figure, the admissible economic performance is satisfied under the specified performance degradation. Besides, the cost of the system reconfiguration is larger than the one of the fault accommodation since the faulty actuator is completely removed in the system reconfiguration. 


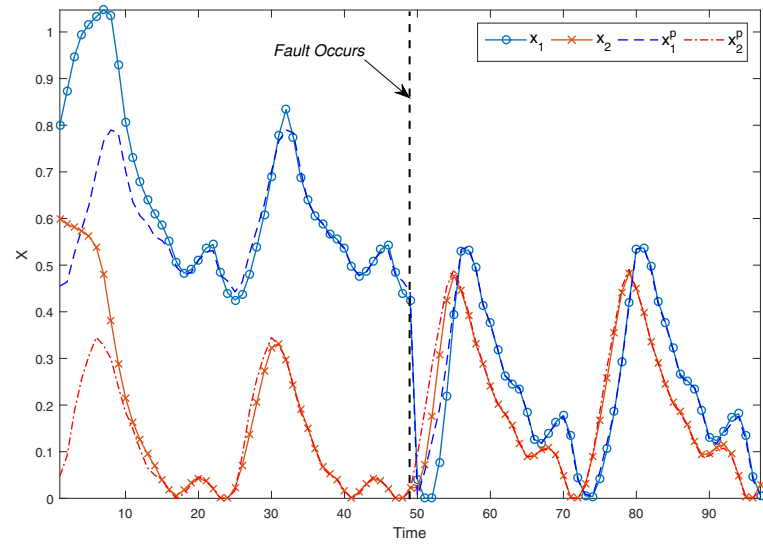

Fig. 4. Fault-tolerant EMPC with System Reconfiguration

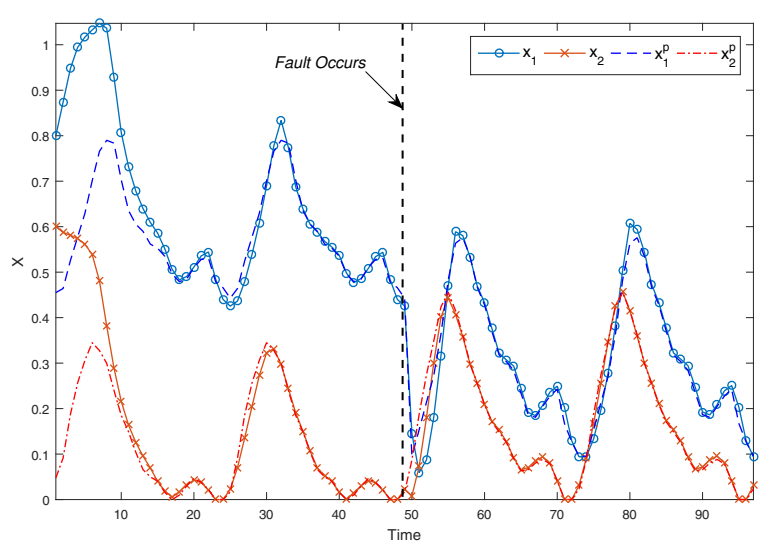

Fig. 5. Fault-tolerant EMPC with Fault Accommodation

\section{CONCLUSIONS}

In this paper, a fault-tolerant periodic EMPC strategy is proposed for DAE systems. An on-line optimization planner is used to find the optimal system steady trajectory. When there are faults in the control system, the performance degradation analysis is carried out by means of the proposed set-based approach for FTC evaluation of DAE systems. After faults occur, if the system can continue running, the steady states with the periodic EMPC strategy are updated. Hence, the on-line planner is appropriate for the inclusion of the fault-tolerant mechanism.

\section{ACKNOWLEDGMENT}

This work has been partially funded by the Spanish Government (MINECO) through the project CICYT ECOCIS (ref. DPI2013-48243-C2-1-R), by MINECO and FEDER through the project CICYT HARCRICS (ref. DPI201458104-R).

\section{REFERENCES}

[1] J. Rawlings and D. Mayne, Model predictive control : theory and design. Madison, Wis. Nob Hill Pub. cop., 2009.

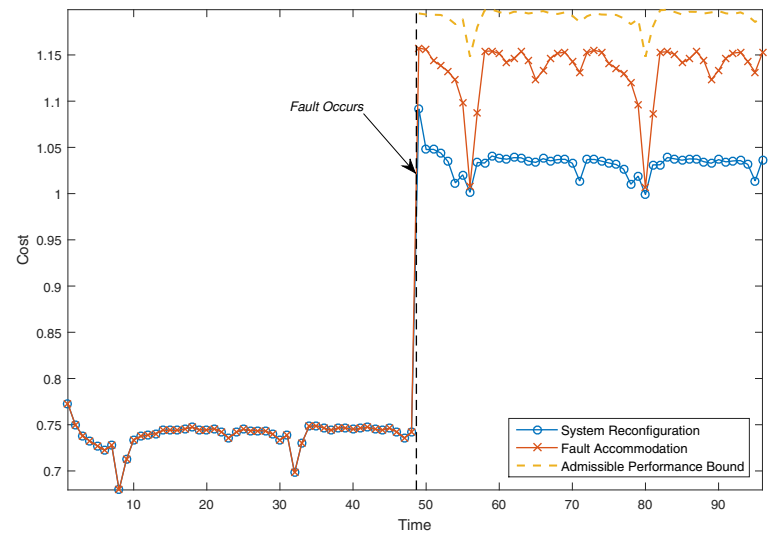

Fig. 6. Economic Costs of Two Fault-tolerant Cases

[2] D. Mayne, "Model predictive control: Recent developments and future promise," Automatica, vol. 50, no. 12, pp. 2967 - 2986, 2014.

[3] L. Biegler, S. Campbell, and V. Mehrmann, Control and Optimization with Differential-Algebraic Constraints. Philadelphia, USA: Society for Industrial and Applied Mathematics, 2012.

[4] Y. Wang, V. Puig, and G. Cembrano, "Economic MPC with periodic terminal constraints of nonlinear differential-algebraic-equation systems: Application to drinking water networks," in European Control Conference, Aalborg, Denmark, 2016, pp. 1013-1018.

[5] J. Mills and A. Goldenberg, "Force and position control of manipulators during constrained motion tasks," Robotics and Automation, IEEE Transactions on, vol. 5, no. 1, pp. 30-46, 1989.

[6] R. Riaza, Differential-algebraic systems: Analytical aspects and circuit applications. World Scientific Publishing Company, 2008.

[7] Y. Wang, V. Puig, and G. Cembrano, "Guaranteed state estimation and fault detection based on zonotopes for differential-algebraic-equation systems," in 3rd International Conference on Control and FaultTolerant Systems, Barcelona, Spain, 2016.

[8] M. Blanke, M. Kinnaert, J. Lunze, M. Staroswiecki, and J. Schröder, Diagnosis and Fault-Tolerant Control. Secaucus, NJ, USA: SpringerVerlag New York, Inc., 2016.

[9] C. Combastel, "A state bounding observer for uncertain non-linear continuous-time systems based on zonotopes," in 44th IEEE Conference on Decision and Control and European Control Conference (CDC-ECC), 2005, pp. 7228-7234.

[10] R. Amrit, J. Rawlings, and D. Angeli, "Economic optimization using model predictive control with a terminal cost," Annual Reviews in Control, vol. 35, no. 2, pp. 178-186, 2011.

[11] D. Angeli, R. Amrit, and J. B. Rawlings, "On average performance and stability of economic model predictive control," IEEE Transactions on Automatic Control, vol. 57, no. 7, pp. 1615-1626, July 2012.

[12] D. Limon, I. Alvarado, T. Alamo, and E. Camacho, "Robust tubebased MPC for tracking of constrained linear systems with additive disturbances," Journal of Process Control, vol. 20, no. 3, pp. 248 260, 2010.

[13] T. J. Broomhead, C. Manzie, R. C. Shekhar, and P. Hield, "Robust periodic economic MPC for linear systems," Automatica, vol. 60, pp. $30-37,2015$.

[14] M. Pereira, D. Mũnoz de la Peña, D. Limon, I. Alvarado, and T. Alamo, "Robust model predictive controller for tracking periodic signals," in European Control Conference, Aalborg, Denmark, 2016.

[15] _ "Application to a large-scale drinking water network of robust mpc for tracking periodic references," in European Control Conference, Aalborg, Denmark, 2016.

[16] D. Limon, M. Pereira, D. Muñoz de la Peña, T. Alamo, and J. Grosso, "Single-layer economic model predictive control for periodic operation," Journal of Process Control, vol. 24, no. 8, pp. 1207 - 1224, 2014.

[17] S. B. Chabane, C. S. Maniu, E. Camacho, T. Alamo, and D. Dumur, "Fault tolerant control approach based on multiple models and set-membership state estimation," in European Control Conference, Aalborg, Denmark, 2016, pp. 1105-1110. 